\title{
ASTROMETRY WITH THE TAUTENBURG SCHMIDT TELESCOPE
}

\author{
K.-G. STEINERT \\ Dresden University of Technology \\ Section of Geodesy and Cartography \\ Lohrmann Observatory \\ Mommsenstraße 13 \\ DDR-8027 Dresden \\ Germany, Democratic Republic
}

\begin{abstract}
The realization of an optimum astronomical reference frame depends on well-defined connection between the systems of optical and radio positions of objects. For this the physics of radio objects are to be studied carefully. Besides this it is necessary to investigate how to get accurate positions of the optical counterparts of these objects as benchmarks for establishing a radio-optical reference frame. As the optical sources are very faint, photographic telescopes with robust light-gathering power are to be used.

For 15 years research work has been done using plates of the 134/200/400 Tautenburg Schmidt telescope for determinations of photographic positions of stars and extragalactic objects. An overview of some special results obtained at Dresden Lohrmann Observatory and Potsdam Central Institute of Astrophysics of the GDR Academy of Sciences will be given.
\end{abstract}

\section{Introduction}

At the Second Astrometric Conference sponsored by the National Science Foundation and the University of Cincinnati, held in Cincinnati, Ohio, 17-21 May 1959, there was a paper by W. Dieckvoss (1960) concerning photographic astrometry with the Bergedorf 32-inch Schmidt telescope.

He presented satisfying results. During the discussion of Dieckvoss' paper there arose some questions connected with the geometry of Schmidt plates which may be disturbed by bending the plate in the plate holder.

In the discussion Herget remarked that in astrometric experiments which he had made with Cleveland and Michigan Schmidt telescopes the residuals of the reduction procedure showed no normal distribution. He found significant systematic effects in various parts of the field.

It seems that because of the contradictory results at the conference there was not adopted any resolution recommending the use of Schmidt telescopes for astrometric purposes.

At the conference on The role of Schmidt telescopes in Astronomy held in Hamburg, 21-23 March 1972, Luyten and La Bonte (1972, p. 35) from Minneapolis spoke about difficulties in the accurate determination of positions for bright stars, using an automatic measuring machine. Moreover, they stated a further improvement of plate reduction could probably be obtained by including more higher-order terms in the solution, but these terms differ from plate to plate. This had been confirmed by Dieckvoss (1972, p. 43). He recommended to use Schmidt telescopes only for small and limited 
fields near the optical axis.

Andersen (1971) came to more optimistic conclusions concerning the Schmidt telescope in astrometry. In his opinion the form of the plate holder (quadratic or circular) plays an important role concerning the mean errors of star positions determined on the plate.

\section{The Lohrmann program}

Sandig (1974) proposed an astrometric program for the Tautenburg Schmidt telescope of the GDR Academy of Sciences, Central Institute of Astrophysics, taking into account:

- some optimistic results of previous astrometric experiences with Schmidt telescopes and the possibility to use the world's largest instrument of this kind;

- that it was necessary to find a way by means of a large telescope to connect the positions of very faint galaxies $\left(18^{\mathrm{m}}\right.$ to $\left.20^{\mathrm{m}}\right)$-among them radio galaxies with optical counterparts-with stars of usual catalogues $\left(9^{\mathrm{m}}\right.$ to $\left.11^{\mathrm{m}}\right)$ and any faint stars;

- that these observations provide the possibility to determine absolute proper motion in a more perfect way than the classical Lick and Pulkovo programs could do with astrographs;

- that it should be possible to improve, for instance, the system of AGK3 and give therefore a contribution to the realization of an optimum astronomical reference frame;

- the possibility to solve problems of stellar astronomy in a fundamental way.

The main idea of Sandig's proposal was to take overlapping plates of belts in declination circles and then later on also in right ascension circles. So he hoped to get a network over the northern sky, including the equatorial zone.

Since astrophysical observations take the main time of the Tautenburg Schmidt it was quite obvious that such a large program could not become realized in a short time. Therefore in 1975 the "Lohrmann Program" was started as a pilot project. The photographic observations of 90 plates with about $20 \%$ overlapping in the $52: 5$ declination zones were finished in 1978 .

\section{Preliminary results of the Lohrmann Program}

Apart from the advantages of Schmidt type instruments for astrometry mentioned above, there were some serious questions to resolve. Böhme and Sandig (1978) published initial results concerning the examination of the instrument.

Having an aperture of $134 \mathrm{~cm}$ of the correction plate and common optical data, the 134/200/400 Tautenburg Schmidt is best fitted for extreme large limiting magnitudes and excellent resolution. The field of $24 \times 24$ square centimeters on the plate equals $3: 1 \times 3: 1$ on the sky. It was stated that the bending effect should not depend upon the size of the field only, but mainly upon the focal distance. Since the focal distance is $400 \mathrm{~cm}$, the bending of $1.2 \mathrm{~mm}$ is comparatively small.

The authors found a star's internal root mean square error of $\pm 0.19\left(9^{\mathrm{m}}\right), \pm 0.09\left(13^{\mathrm{m}}-15^{\mathrm{m}}\right)$, and \pm 0 ". $13\left(17^{\mathrm{m}}\right)$ in the center of the plate and $\pm 0.11\left(13^{\mathrm{m}}-15^{\mathrm{m}}\right), \pm 0$ ". $15\left(17^{\mathrm{m}}\right)$ in the outer parts of the plate. On the other hand, the internal root mean square error of positions of galaxies derived from double observations on one plate for 48 to 75 measured objects as a mean value of four plates was obtained as \pm 0.24 and \pm 0 ". 36 depending on the quality of the images.

To avoid the effect of magnitude equations between bright stars $\left(9^{\mathrm{m}}-10^{\mathrm{m}}\right)$ and faint galaxies $\left(17^{\mathrm{m}}-\right.$ 
$18^{\mathrm{m}}$ ) in the Lohrmann program there was used a chromatic prism in eccentric position mounted in front of the correction plate. So an object on the sky yields one image $A$ at the plate produced from the whole aperture of the telescope, and a deflected one $B$ by the prism about 4 magnitudes fainter and about 30" transversally displaced, corresponding to the eccentric position of the prism and its refracting angle. The long exposure ( $A, B$ images) was 20 minutes. A second, shorter exposure (20$30 \mathrm{sec}$ ) on the same plate after displacement of the optical axis about $1 \mathrm{arcmin}$ in declination yielded an image $C$, in magnitude corresponding to $B$ with full aperture, and $D$ about $4^{\mathrm{m}}$ fainter than $B$ and $C$ through the prism.

That means that a bright object leaves on the plate four images: $A$ brighter than $B$ and $C$ by 4 magnitudes and $D$ fainter by 4 magnitudes than the latter ones. Presuming a correct mutual geometry of the images $A . . . D$ it should be possible to reduce all images to the position of, for instance, $A$. At that time (Böhme and Sandig 1978) no problem seemed to exist in reduction of the plates. The closed adjustment of the whole declination circle was thought to be done with AGK3 as a reference catalogue.

The totality of selected stars was to cover the zenith zone of Potsdam PZT stars and AGK3 stars as reference stars. Moreover, faint stars of $16^{\mathrm{m}}-18^{\mathrm{m}}$ should be selected to establish direct connection with extragalactic objects.

Since the time of designing the program there arose a multitude of questions and problems of very different kind. They were investigated in Dresden Lohrmann Observatory as well as in PotsdamBabelsberg Central Institute of Astrophysics.

Some results will be presented in the following sections.

\section{Special investigations of some astrometric properties of the Tautenburg Schmidt}

\subsection{THE PLATE HOLDER}

Andersen (1971) pointed out that a circular plate holder guarantees smaller forced deformations than a quadratic one. Böhme (1983) showed, after a thorough investigation of both types of plate holders, that there are no significant systematic effects in the positions for either the circular or the quadratic plate holders of the Tautenburg instrument. So the second epoch of the Lohrmann program will be taken with the quadratic plate holder, was was done for the first epoch.

\subsection{INFLUENCE OF EXCHANGING THE MIRROR OF THE TELESCOPE}

After 25 years successful work of the Karl-Schwarzschild-Observatorium, founded in 1960, the glass mirror of the Schmidt telescope was replaced by a mirror consisting of the glass-ceramic material Sitall (Marx 1986).

The glass mirror has had an excellent optical quality, but the glass-mass showed a temperature dependency. A temperature difference of $2^{\circ} \mathrm{C}$ between the outer and inner surfaces of the mirror yielded an image size of 1".5-2:"0, which is on the order of the seeing disc. For Sitall this occurs only for a difference of $100^{\circ} \mathrm{C}$.

The astrometric qualities of the new Sitall objective were tested in the course of a student's research work. Two plates, taken in 1984 July with the glass objective, were compared with two plates of the same field in the sky, taken in 1985 July with the Sitall mirror. The result was a very good agreement of the positions in both groups of exposures (1984/1985) for stars fainter than about 11 mag. Only brighter stars on plates with the Sitall mirror show larger errors and significant variations of positions, 
because the images of these objects at the plate show noncentric aureolas.

\subsection{Plate Reduction models}

Numerous calculations have been carried out using different models for the plate reduction. At first, all plates of the 90 fields of the Lohrmann Program were reduced using the orthogonal transformation of the measured plate coordinates $x, y$ into the tangential coordinates $X, Y$ with four plate constants:

$$
\begin{aligned}
& X-x=A x+B y+E \\
& Y-y=-B x+A y+F .
\end{aligned}
$$

This simple model was used because a non-rectangularity of the guide rulers of the measuring machine (ASCORECORD 3 DP/ASCOREMAT) is considered by the software.

In the course of time at Dresden Lohrmann Observatory it was understood (Witschas 1987) that for a routine program it is more useful to reduce the data with an affine model of the kind

$$
\begin{aligned}
& X-x=A x+B y+E \\
& Y-y=C x+D y+F
\end{aligned}
$$

with 6 plate constants.

Dick (1989) advocates on the basis of a development by Hirte (1988) a reduction method which uses the mathematical procedure of stepwise regression. That means, in a polynomial of second or third order, that all plate constants became tested for significance. If significance is probable, the respective plate constants are used for reduction of the plates. It is known since 1972 (Luyten and La Bonte) that the constants differ from plate to plate-and therefore the significances do also.

The author is of the opinion that the method of stepwise regression is a good means to get the best mathematical model for adapting the positions on a plate to the system of standard coordinates. But for the necessity of uniformity and homogeneity in a program with a large number of plates $(e . g$., the Lohrmann Program) it seems to be more useful to use a unique reduction model such as, for instance, the affine one under the conditions of a well-adjusted instrument and taking into account the effect of bending.

\subsection{GEOMETRY OF THE SCHMIDT PLATES}

As was confirmed in the previous section, the method of stepwise regression in reduction of plates is a very good mathematical procedure. An imperfection of this method is that physical arguments for the selection of plate constants depending on the results of statistical tests are not known.

Witschas (1983) proposed to use the method of least squares collocation for the analysis of irregular systematic errors on Tautenburg plates. By this method it should be possible to avoid smearing effects which are introduced by any number of plate constants handling the plate as a geometrical unit.

Witschas (1987) found the root mean square errors of unit weight $\sigma_{0}$ as shown in Table 1. The better modelling of the plate geometry by about $20 \%$ with collocation is obvious. (The large values in Table 1 arise from the catalogue errors of the AGK3). Unfortunately, the collocation analysis must be done for every single plate. In this way, the collocation has the same imperfection as the stepwise regression.

Another serious, not fully answered problem, is how to refer the $A, B, C$ and $D$ images of an object 
to a single position, so that these reduced positions represent a geometric uniform model of the photographed field.

Böhme (1989) found a general possibility to reduce the images $A$ (exposure $20 \mathrm{~min}$ ) and $C$ (exposure $30 \mathrm{sec}$ ) of objects over the plate into one homogeneous system. He did not use equatorial coordinates, but only the differences of positions $A$ versus $C$ images on the plate. Thus the differences could be declared using only components of translation, rotation and inclination. The transformation into equatorial coordinates was then done in a common manner.

The mean values of the calculated and measured differences of $C$ images after transformation to the $A$ positions are on the order of measuring accuracy, namely 1.1 to 2.6 micrometers.

Table 1. Comparison of adjustments with plate constants and with collocation

\begin{tabular}{lllll} 
Plate No & \multicolumn{2}{l}{ plate constants } & \multicolumn{2}{l}{ collocation } \\
& $\sigma_{\text {ox }}$ & $\sigma_{\text {oy }}$ & $\sigma_{\text {ox }}$ & $\sigma_{\text {oy }}$ \\
4501 & \pm 0.393 & \pm 0.452 & $\pm 0 " .252$ & \pm 0.367 \\
5182 & \pm 0.376 & \pm 0.483 & \pm 0.289 & \pm 0.370
\end{tabular}

\section{Some results and future contributions}

The main fields for application of the Tautenburg Schmidt telescope in astrometry are

- the determinatin of relative positions of objects on the sky

- the determination of absolute proper motions of the stars with respect to galaxies

- orientation of a future HIPPARCOS system with optical and radio sources.

In these fields a series of contributions were written mainly by Potsdam authors.

The determination of proper motions of different objects was done by Schilbach (1982), and Scholz and Rybka (1988). Positions of extragalactic objects were determined by Dick et al (1986). A proposal for orientation of the HIPPARCOS system was made by Dick et al. (1987) and by Yatsenko et al. (1987).

An essential task for the future is the connection between optical and radio reference frames. The success of this depends on a well defined relation between the physically very different objects in radio and optical radiation. Hitherto there is no definitive evidence that the positions of extragalactic objects, relevant to solve this problem, are independent from the frequency of their radiation.

Without any doubt the compiled radio positions catalogue CC by Walter (1989) will be the basis of future celestial reference frames. However, until now it is not satisfactorily resolved that the origin of the radio right ascensions is either 3C273, a radio galaxy with variations of its structure, or Beta Persei (Algol), a variable star.

It is quite certain that after the observations of the second epoch of the Lohrmann Program there will be a good foundation to determine exact absolute proper motions of stars of any magnitude in the declination belt of $52^{\circ} .5$. 


\section{References}

Anderson, J.: 1971, "The Schmidt telescope as an astrometric instrument", Astron. Astrophys. 13, $40-45$.

Böhme, D. and Sandig, H.U: 1978, "Present status of Lohrmann Program", in F.V. Prochazka and R.H. Tucker (eds.) Modern Astrometry, University Observatory, Vienna, pp. 538-542.

Böhme, D.: 1983, "Einfluß des Kassettentyps beim 2-m-Schmidt-Spiegel auf die Genauigkeit astrometrischer Positionen", in K.-G. Steinert (ed.), VI. Internationales Lohrmann-Kolloquium Geodätische Astrometrie, Mitt. Lohrmann-Obs. No. 51, pp. 112-114 (Wiss. Zeitschrift TU Dresden 33.6).

Böhme, M.: 1989, "Geometrie von Sternpositionen auf Tautenburger Schmidtplatten mit unterschiedlicher Belichtungszeit (A/C-Bilder)”, Diploma thesis, Tech. Univ. Dresden, Lohrmann Obs.

Dick, W.R., et al.: 1986, "Positions of extragalactic objects in the vicinity of M3 and M31 (5C3 radio field) and in the 5C1 radio field", Astron. Nachr. 307, 85-88.

Dick, W.R. et al.: 1987, "A programme to derive from terrestrial observations the rotation of the HIPPARCOS system with reference to the inertial system", Astron. Nachr. 308, 211-216.

Dick, W.R.: 1989, "Die astronomischen Eigenschaften des Tautenburger Schmidt-Teleskops und seine möglichen Beiträge zur Realisierung eines Inertialsystems", Doctor thesis, GDR Acad. Sciences, Forschungsbereich Geo- und Kosmowissenschaften.

Dieckvoss, W.: 1960, "The Bergedorf 32-inch conventional Schmidt telescope as an astrometric instrument", Astron. J. 65, 214-217.

Dieckvoss, W.: 1972, "Computational solution for positions on whole Schmidt plates", in U. Haug (ed.) The role of Schmidt telescopes in Astronomy, ESO/SRC/Hamburger Sternwarte Bergedorf, pp. 39-43.

Hirte, S.: 1989, "Reduction methods for the astrometric analysis of Schmidt plates", in K.-G. Steinert (ed.) VII. Internationales Lohrmann-Kolloquium Geodätische Astrometrie, Mitt. LohrmannObs. No. 56, pp. 15-16 (Wiss. Zeitschrift TU Dresden 38.2)

Luyten, W.J. and La Bonte, A.E.: 1972, "Astrometry with Schmidt telescopes" vide Dieckvoss (1972) pp. 33-38.

Marx, S.: 1986, "Jenaer 2-m-Spiegelteleskop in Tautenburg erhielt neuen Spiegel", Jenaer Rundschau 31, 132-133.

Sandig, H.U.: 1974, "Zur systematischen Verbesserung von Positionen und zur Bestimmung absoluter Eigenbewegungen mit der Tautenburger Schmidt-Kamera", in H. Kautzleben and E. Buschmann (eds.) 2nd International Symposium Geodesy and Physics of the Earth, Proceedings Part 1, 105-111.

Schilbach, E.: 1982, “Absolute Eigenbewegungen ausgewählter blauer Objekte in der Nähe des galaktischen Nordpols", Astron. Nachr. 303, 335-340.

Scholz, R.D.: 1988, "Comparison of three catalogues of absolute proper motions of stars in the M33 field”, Astron. Nachr. 309, 47-52.

Walter, H.G.: 1989, “A compilation catalogue of positions of extragalactic radio sources", Astron. Astrophys. 210, 455-461.

Witschas, Ch.: 1983, “Kollokation auf Schmidtplatten”, vide Böhme (1983), pp. 75-76.

Witschas, Ch.: 1987, "Untersuchungen zum astrometrischen Informationsgehalt von Tautenburger Schmidtplatten", Doctor thesis, Tech. Univ. Dresden, Fakultät BWF.

Yatsenko, A.I. et al.: 1987, "The connection of the HIPPARCOS reference system to extragalactic objects by photographic astrometry", Astron. Nachr. 308, 319-322. 